\title{
Successful Management of Two Patients with Intracranial Hemorrhage due to Carpet Viper (Echis ocellatus) Envenomation in a Limited-Resource Environment
}

\author{
Jordan M. Benjamin, EMT-P, NRP ${ }^{1,2}$; Jean-Philippe Chippaux, MD, $\mathrm{PhD}^{1,3,4}$; Bio Tamou-Sambo, $\mathrm{MD}^{5,6}$; \\ Olouchégoun Cardinal Akpakpa, $\mathrm{MD}^{7}$; Achille Massougbodji, $\mathrm{MD}^{5}$ \\ ${ }^{1}$ Asclepius Snakebite Foundation, Seattle, WA; ${ }^{2}$ Department of Biology, Whitman College, Walla Walla, WA; ${ }^{3}$ Center for the Study and Research \\ of Malaria Associated with Pregnancy and Childhood (Cerpage), Cotonou, Benin; ${ }^{4}$ French Institute of Research for Development, Marseille, \\ France; ${ }^{5}$ Département de chirurgie et spécialités, Université Parakou, Parakou, Benin; ${ }^{6}$ Hôpital Evangelique Bembèrèkè, Bembèrèkè, Benin; \\ ${ }^{7}$ Centre de santé communal de Nikki, Parakou, Bénin
}

\begin{abstract}
The West African carpet viper (Echis ocellatus) causes more deaths than any other snake in subSaharan Africa. Carpet viper envenomations are characterized by a venom-induced consumption coagulopathy and systemic bleeding syndrome, in addition to local symptoms of painful progressive swelling and tissue destruction. The highest mortality rate is seen in the final stages of the syndrome, which typically ends with fatal internal bleeding or hemorrhagic shock. We present 2 cases of E ocellatus envenomation with intracranial hemorrhage seen at a rural hospital in Bembèrèkè, Benin, and describe the successful management of these patients in a limited-resource setting. In one case the patient was treated with an ineffective Indian-made antivenom before evaluation by the authors and continued to deteriorate until she was treated with effective antivenom $10 \mathrm{~d}$ after the bite. In both cases lumbar puncture was performed for diagnostic or therapeutic purposes with good effect, and both patients made full recoveries without sequelae. These cases demonstrate the remarkable ability of high-quality antivenoms to reverse life-threatening envenomations even in the final stages of the hemorrhagic syndrome and illustrate the dangers posed by low-quality antivenoms that have flooded the market in the developing world.
\end{abstract}

Keywords: snakebite, snake envenomation, neglected tropical disease, venom-induced consumption coagulopathy, antivenom, whole blood clotting test

\section{Introduction}

The West African carpet viper (Echis ocellatus; Figure 1) is responsible for more bites and deaths than any other snake in sub-Saharan Africa. ${ }^{1-5}$ The combination of its diminutive size, unusually low incidence of dry bites, and ability to thrive in human-disturbed habitats including farms and villages causes frequent encounters with humans. Procoagulant components of carpet viper venom act at various levels of the clotting cascade to produce a venom-induced consumption coagulopathy (VICC), and other molecules known as hemorrhagins directly attack the vascular endothelium to promote

Corresponding author: Jordan Benjamin, Asclepius Snakebite Foundation, Seattle, WA, 98107; e-mail: jordan@ @nakebitefoundation.org.

Submitted for publication September 2018.

Accepted for publication April 2019. bleeding. ${ }^{5-8}$ Carpet viper envenomation syndrome is therefore characterized by a devastating combination of incoagulable blood and widespread bleeding in addition to the painful progressive swelling and tissue destruction that is typical of most viper envenomations. The classic progression of the syndrome is outlined in the clinical staging table (Table 1). The later stages are associated with significantly higher mortality, with the highest mortality in hemorrhagic snakebites seen in patients with intracranial hemorrhage (ICH). ${ }^{4,9}$ We present 2 cases of E ocellatus envenomation with ICH treated at a rural hospital in Bembèrèkè, Benin, and describe the diagnosis and successful management of these patients in a limited-resource setting. Both patients were treated during the clinical trial of Antivipmyn Africa in northern Benin, which was cleared by the ethics committee of the Faculty of Health Sciences of the University of Abomey-Calavi, 


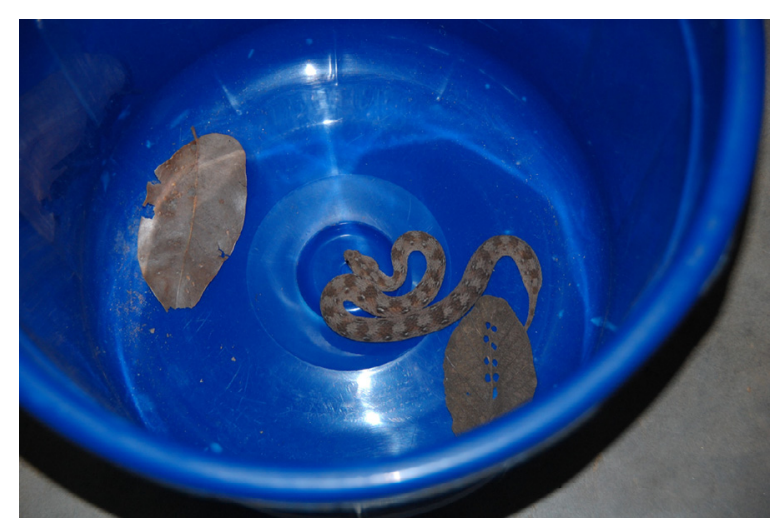

Figure 1. An adult carpet viper (Echis ocellatus) is small enough to hide under a single leaf and often remains unnoticed by local subsistence farmers until it is too late.

Benin in June 2005 and renewed in June 2013 (14/06/ 2013 / EC / FSS / UAC). Informed consent was obtained from patients and their families in all cases.

\section{Case 1}

A 12-y-old Beninese girl (Patient 1) presented to the hospital in Bembèrèkè during the rainy season in 2012 after sustaining an E ocellatus bite to her right foot $4 \mathrm{~d}$ earlier $\left(D_{4}\right)$. At this time the authors had not yet arrived at the hospital, so information from the initial period of her care was obtained retrospectively from her chart. On arrival, the patient presented with painful edema past the ankle and active gingival bleeding with a hematocrit of $24 \%$ and temperature of $37^{\circ} \mathrm{C}$. She was treated with 2 vials of Asna Antivenom C (AV-C; Bharat Serums and Vaccines Ltd, Mumbai, India) and immediately developed a fever of $39^{\circ} \mathrm{C}$, which was reduced with acetaminophen and metamizole, an anti-inflammatory analgesic that is no longer available in the United States owing to a risk of agranulocytosis but which remains widely used in sub-Saharan Africa. She was prescribed $1000 \mathrm{mg}$ of acetaminophen 3 times a day and $500 \mathrm{mg}$ cloxacillin 4 times a day. On $\mathrm{D}_{6}$ she developed a new onset of hematemesis and epistaxis; examination revealed pale mucosa, blood pressure of 70/40 mm Hg, and hematocrit of $10 \%$. She was treated with 2 additional vials of AV-C, 2 units of whole blood ( $450 \mathrm{~mL}$ each), 1 ampoule containing $500 \mathrm{mg}$ tranexamic acid by IV push, 1 ampoule of vitamin $\mathrm{K}$ (dosage unspecified) given subcutaneously, and $500 \mathrm{mg}$ tablets of both iron and vitamin $\mathrm{E}$ to be taken 4 times a day. On $\mathrm{D}_{8}$ her hematocrit was $15 \%$, but active hematemesis had resumed. She was given another $500 \mathrm{mg}$ ampoule of tranexamic acid, and 1 additional unit of whole blood was transfused.

On $\mathrm{D}_{10}$ one of the authors arrived at the hospital and examined the patient for the first time at $\mathrm{H}_{0}$ (hour 0; hours since initial assessment by the authors). Vital signs were as follows: blood pressure $90 / 50 \mathrm{~mm} \mathrm{Hg}$; heart rate 65 beats $\mathrm{min}^{-1}$; temperature $37.2^{\circ} \mathrm{C}$; respiration 28 breaths $\cdot \mathrm{min}^{-1}$ and labored; skin pale, cool, and clammy; and pupils equal and reactive to light. Hematocrit was now $23 \%$ after the multiple transfusions. There was no visible bleeding on examination, but the whole blood clotting test (WBCT) showed completely incoagulable blood at $30 \mathrm{~min}\left(\mathrm{WBCT}_{30}\right)$. WBCT evaluation at $20 \mathrm{~min}$ $\left(\mathrm{WBCT}_{20}\right)$ was not taken in this patient. Physical examination revealed signs of elevated intracranial pressure (ICP) including prostration, nuchal rigidity, positive Brudzinski's sign, photophobia, severe pain on palpation of the spine, severe headache and neck pain described as "pressure," nausea, and intermittent episodes of obtundation. There were no focal neurological findings on careful examination despite the patient's altered mental status. The patient was crying out from severe pain, and the only analgesics available at the time (IV acetaminophen and metamizole) had proven ineffective. The combination of her history, clinical presentation, positive $\mathrm{WBCT}_{30}$, and the pathophysiology of Echis envenomation were strongly suggestive of an ICH.

The patient was immediately given 2 vials of Antivipmyn Africa (Bioclon, Mexico) in $10 \mathrm{~mL}$ of saline by direct IV push over $3 \mathrm{~min}$. The primary concern was that the patient would not survive long enough for the antivenom to take effect in light of her rapidly

Table 1. Simplified staging system for charting clinical progression of the hemorrhagic syndrome of envenomation in subSaharan Africa.

\begin{tabular}{ll}
\hline Stage & Coagulopathy \pm bleeding \\
\hline Stage 0 & Coagulopathy without spontaneous bleeding \\
Stage 1 & Persistent atraumatic bleeding from the bite wound $>1 \mathrm{~h}$ \\
Stage 2 & Bleeding from old cuts and wounds elsewhere on patient \\
Stage 3 & Spontaneous bleeding from healthy mucosa (eg, gingival bleeding, epistaxis) \\
Stage 4 & Externalization of internal bleeding (eg, hematemesis, melena) \\
Stage 5 & Intracranial, intra-abdominal, or retroperitoneal bleeding; hemorrhagic shock \\
\hline
\end{tabular}


deteriorating condition. After consultation between colleagues and in consideration of the lack of resources available to manage her rising ICP, the decision was made to attempt a therapeutic lumbar puncture to relieve some of the pressure, gain time for the antivenom to work, and permit additional antivenom to be given if the bleeding persisted. Lumbar puncture was performed at $\mathrm{H}_{3}$ and revealed dark red blood in the cerebrospinal fluid (CSF), confirming the diagnosis of ICH (Figure 2). It was not possible to obtain an opening pressure because of a lack of necessary equipment at the hospital. Several vials of bloody CSF were collected, the needle was removed, and direct pressure was applied to the puncture site for several minutes. The confirmation of active bleeding allowed an additional 2 vials of Antivipmyn to be administered for persistence of active hemorrhage at this time as per the treatment protocol, which is described elsewhere. ${ }^{10-12}$

The patient was placed in a position of comfort with the head of the bed elevated 30 degrees, and ice was placed on her head and neck. A marked decrease in her level of distress was observed immediately after the procedure; she was able to sleep for the first time in over 24 h. At $\mathrm{H}_{8}$ the authors were able to obtain $60 \mathrm{mg}$ of furosemide, which was given IV, and $10 \mathrm{mg}$ of diazepam, which was given intramuscularly to provide sedation in the absence of effective analgesia. By $\mathrm{H}_{12}$ her pain had improved slightly, and $\mathrm{WBCT}_{30}$ improved from no clot to a partial, friable clot. Her overall condition remained largely unchanged, but she appeared to have stabilized and was no longer deteriorating. Her blood pressure at $\mathrm{H}_{12}$ remained low, but her heart rate increased to 75 to

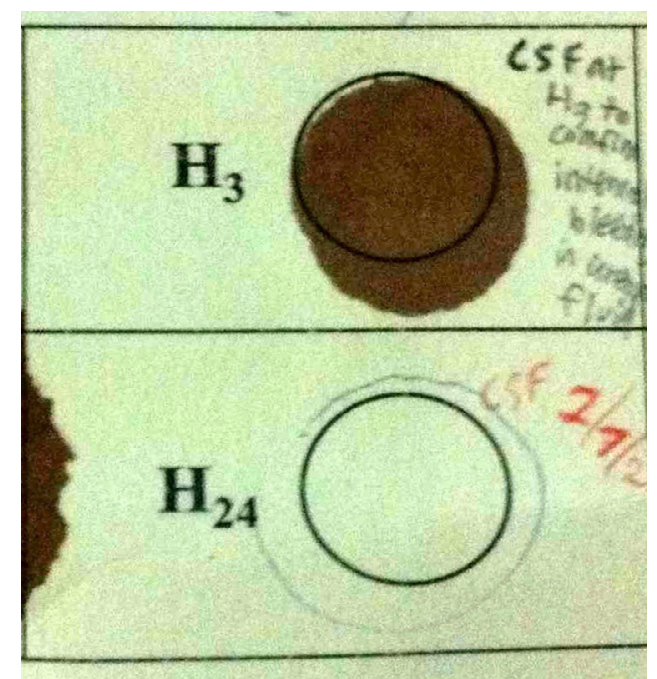

Figure 2. Cerebrospinal fluid (CSF) samples collected from Patient 1 at $\mathrm{H}_{3}$ and at $\mathrm{H}_{96}$.
80 beats $\cdot \mathrm{min}^{-1}$, respirations decreased to 20 to 24 breath$\mathrm{s} \cdot \mathrm{min}^{-1}$, and mental status had improved. She continued to improve steadily over the next $2 \mathrm{~d}$. $\mathrm{WBCT}_{30}$ remained abnormal at $\mathrm{H}_{24} / \mathrm{D}_{11}$ and $\mathrm{H}_{48} / \mathrm{D}_{12}$, but stability of the clot increased and pain decreased consistently during that time. By $\mathrm{H}_{48} / \mathrm{D}_{12}$ her pain had improved dramatically and vital signs were within normal limits. At $\mathrm{H}_{96} / \mathrm{D}_{14}$ the $\mathrm{WBCT}_{30}$ normalized and a repeat lumbar puncture showed clear CSF (Figure 2), confirming complete resolution of the hemorrhagic syndrome. She received a tetanus vaccine and was discharged from the hospital with a complete recovery and no neurological deficit $15 \mathrm{~d}$ after the bite and $120 \mathrm{~h}$ after initial treatment with Antivipmyn Africa.

\section{Case 2}

An 18-y-old Beninese man (Patient 2) presented to the hospital in Bembèrèkè during the rainy season in 2012 reporting an E ocellatus bite to his left foot that occurred $4 \mathrm{~d}$ earlier. Examination upon arrival at $\mathrm{H}_{0} / \mathrm{D}_{4}$ revealed persistent bleeding from 2 fang marks, prominent edema ending $5 \mathrm{~cm}$ ( 2 in) below the left knee, bleeding from the gingival sulci, sublingual hematoma, dysphagia, inguinal lymphadenopathy, and tenderness on palpation of edematous submandibular and temporomandibular tissues. Pain was reported upon palpation of the length of the spine, the worst of which was noted from the upper cervical vertebrae into the suboccipital triangle. Severe headache, nuchal rigidity, and Kernig's sign were documented without fever, and a presumptive diagnosis of ICH was made. The patient was distressed but had appropriate mentation, and no focal neurological findings were identified on examination. Blood pressure was 150/110 mm $\mathrm{Hg}$; heart rate 90 beats $\min ^{-1}$, weak, and thready; and respiratory rate was 30 breaths. $\mathrm{min}^{-1}$, rapid, and shallow. Skin was warm and dry, and mucosal membranes were pale. Bilateral conjunctival hemorrhage was noted, although pupils remained equal, responsive, and reactive to light. $\mathrm{WBCT}_{20}$ and $\mathrm{WBCT}_{30}$ showed completely incoagulable blood, and hematocrit was $21 \%$.

The patient was given 2 vials of Antivipmyn at $\mathrm{H}_{0} / \mathrm{D}_{4}$ and received an additional 2 vials of Antivipmyn at $\mathrm{H}_{3}$ after lumbar puncture confirmed the diagnosis of an active ICH (Figure 3). As in the first case, no equipment was available to obtain an opening pressure. He improved rapidly after the second dose of antivenom at $\mathrm{H}_{3}$. $\mathrm{WBCT}_{20}$ and $\mathrm{WBCT}_{30}$ resolved by $\mathrm{H}_{6}$, and a repeat lumbar puncture at $\mathrm{H}_{18}$ yielded clear CSF indicating complete resolution of the $\mathrm{ICH}$ (Figure 4). At $\mathrm{H}_{24} / \mathrm{D}_{5}$ he reported that the headache had returned when seated forward; it improved with ice and elevation of the head of the bed to 30 degrees. 


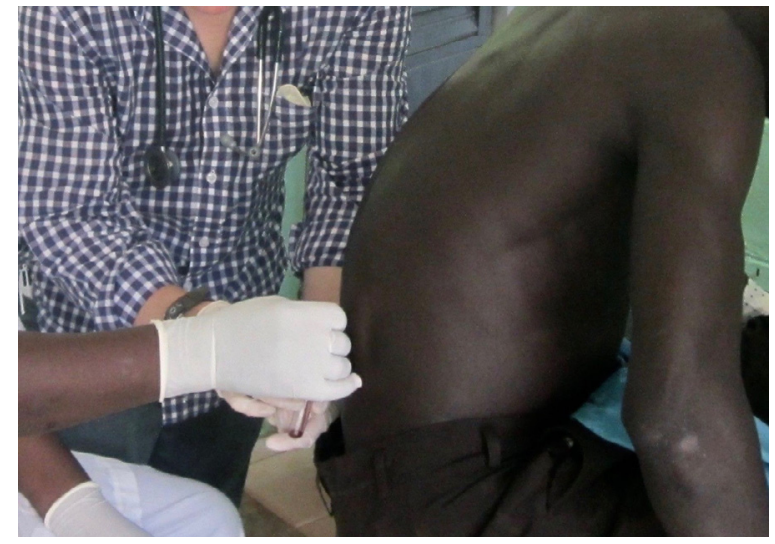

Figure 3. Collection of cerebrospinal fluid (CSF) by lumbar puncture from Patient 2 at $\mathrm{H}_{3}$ before his second round of antivenom treatment. Note the color of the CSF draining into the tube.

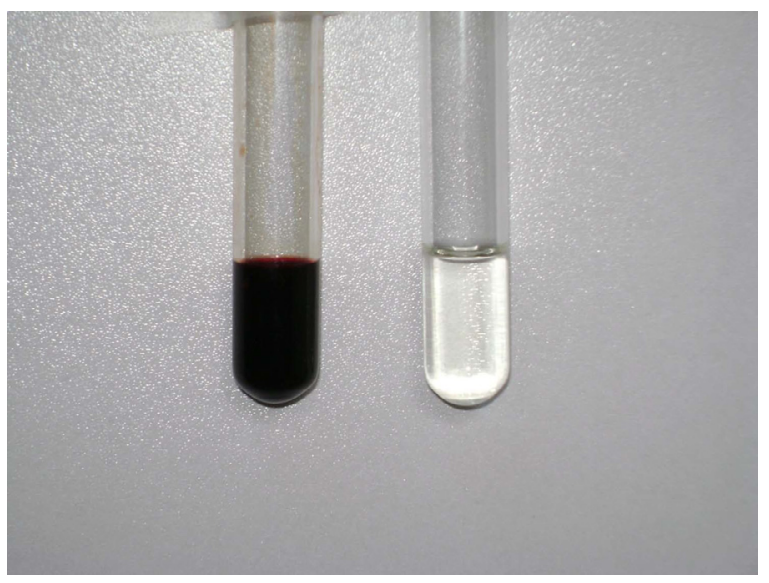

Figure 4. Cerebrospinal fluid obtained from Patient 2 by lumbar puncture at $\mathrm{H}_{3}$ before the second round of antivenom treatment (L) and $18 \mathrm{~h}$ after antivenom treatment $(\mathrm{R})$.

Both $\mathrm{WBCT}_{20}$ and $\mathrm{WBCT}_{30}$ remained normal at $\mathrm{H}_{24}$. At $\mathrm{H}_{27} \mathrm{WBCT}_{30}$ showed an abnormal partial clot and headache had worsened slightly, but we did not administer additional antivenom owing to the discrepancy between WBCT results, overall improvement of the patient, and absence of blood in the CSF sample obtained several hours earlier. However, by $\mathrm{H}_{48} / \mathrm{D}_{6}$ both $\mathrm{WBCT}_{20}$ and $\mathrm{WBCT}_{30}$ revealed abnormal, friable clots, and the headache persisted even in previous positions of comfort. Based on the agreement now observed between $\mathrm{WBCT}_{20}$ and $\mathrm{WBCT}_{30}$ and the worsening symptoms, we administered 2 additional vials of Antivipmyn at $\mathrm{H}_{48}$ for a suspected resumption of ICH. The patient progressively improved over the next several days. Permanent resolution of $\mathrm{WBCT}_{20}$ and $\mathrm{WBCT}_{30}$ was observed at $\mathrm{H}_{120} / \mathrm{D}_{9}$, and he was given a tetanus vaccination and discharged $36 \mathrm{~h}$ later on $\mathrm{D}_{10.5}$ after a complete recovery without sequelae.

\section{Discussion}

The management of snakebite patients with ICH is one of the most difficult areas of clinical toxinology and is associated with a very high mortality rate, even in wellequipped modern hospitals. A case study of snakebite patients with ICH in Australia found that 5 of 6 patients died despite antivenom therapy and intensive care. ${ }^{9}$ Studies in Nigeria and Cameroon reported fatalities in 7 of 10 total cases of suspected or confirmed ICH secondary to $E$ ocellatus envenomation. ${ }^{3,5}$ The presence of CNS symptoms indicating ICH was the strongest predictor of mortality in a retrospective review of over 6687 snakebite patients, predominately $E$ ocellatus envenomations. ${ }^{4}$ The Australian study identified advanced age and medical history of hypertension as risk factors for ICH from snakebite, but neither our patients nor the majority of those reported in other papers describing ICH secondary to Echis envenomation fit either of these criteria. The mechanism of ICH may differ between Echis and the Australasian elapids, where comorbidities seem to play a key role in its development. One study examining the effect of venom from the Palestine or Israeli saw-scaled viper (Echis coloratus) venom on brain capillaries in mice found that envenomation resulted directly in a breakdown of the blood-brain barrier. ${ }^{13}$

Remarkably, both of our patients not only survived but made complete recoveries despite the long delays to effective antivenom, severity of their conditions, and limited resources available at the hospital. These cases serve as an important reminder that it is never too late to administer antivenom to a patient with an active envenomation. The secondary recurrence of VICC is a strong indicator of active venom in the bloodstream and signals the need for additional antivenom in cases of hemorrhagic syndrome. ${ }^{14}$ Performing a delayed double reading of WBCT results at both 20 and $30 \mathrm{~min}$ is a simple but effective means of identifying the onset, persistence, or recurrence of VICC in austere environments and is helpful in guiding the decision to give another dose of antivenom. ${ }^{10}$ In the case of the second patient, the abnormal WBCT $_{30}$ at $\mathrm{H}_{27}$ would have led to earlier treatment if we had not waited until the abnormal $\mathrm{WBCT}_{20}$ at $\mathrm{H}_{48}$ to confirm the need for additional antivenom.

The decision to perform lumbar puncture in the presence of VICC carried significant risks, including spinal hematoma, catastrophic hemorrhage, and uncal herniation; however, under the extreme circumstances we believed that it was justified given the likely alternative of an excruciating death without analgesia if decisive action was not taken. The procedure would be absolutely contraindicated in the presence of focal neurological deficits owing to increased risks of accelerating 
herniation. Four tubes of CSF were collected from each patient, and a total of approximately $30 \mathrm{~mL}$ was drained during each procedure. To verify that the striking appearance of the initial samples obtained by lumbar puncture resulted from ICH rather than a traumatic tap, all tubes of CSF were visually inspected for xanthochromia and red blood cell clearing using hematocytometers and by experienced technicians in the hospital laboratory. ${ }^{15-17}$ There was no decline in red blood cells between the first and fourth tubes collected from either patient during the initial lumbar puncture, and xanthochromia was reported in all samples obtained before and after antivenom treatment. A rapid reduction in ICP may have proven particularly important for patient 1 by delaying the impending herniation her rapidly deteriorating condition indicated and securing valuable time for the antivenom to work. Although it is impossible to know whether a reduction in ICP contributed to the survival of these patients, there were no complications from lumbar puncture in either case, and both recovered fully despite the poor prognosis of ICH in snakebite. Furthermore, the decrease in agitation observed in Patient 1 immediately after the procedure would suggest that it was of some benefit. To our knowledge this is the first time that therapeutic lumbar puncture has been used as a tool for diagnosis and stabilization of active ICH secondary to snake envenomation in austere environments. In both cases, confirmation of an ongoing ICH by lumbar puncture at $\mathrm{H}_{3}$ allowed us to administer additional doses of antivenom in a timely and accurate manner according to the treatment protocol we were investigating. Other methods for temporarily reducing elevated ICP in remote settings include the use of a burr hole or endotracheal intubation with capnography-guided hyperventilation to maintain end-tidal carbon dioxide within a range of 30 to $35 \mathrm{~mm} \mathrm{Hg}$, but neither option was available to us. Any interventions to reduce ICP in these patients should be considered stabilizing measures with the ultimate goal of keeping the patient alive until appropriate antivenom can resolve the underlying issue.

It is worth noting that $\mathrm{AV}-\mathrm{C}$, which proved completely ineffective in halting the hemorrhagic syndrome in Patient 1, has a well-documented history of failure in sub-Saharan Africa. AV-C is produced using venom from the Indian saw-scaled viper (Echis carinatus) and marketed as an African antivenom despite a demonstrated lack of efficacy against African Echis species in both clinical and preclinical studies. ${ }^{18,19}$ In 2008 a rural hospital in Ghana reported a 6-fold increase in mortality after switching from high-quality FAV-Afrique (FAV-A; Aventis Pasteur, Lyon, France) to the less expensive AV-C. ${ }^{20}$ Any difference in cost was negated by the large amount of AV-C required during the study to compensate for its lower efficacy. More than half (56\%) of all patients treated with AV-C required additional doses of antivenom compared to less than one quarter $(22 \%)$ of those treated with FAV-A. In a smaller subset of patients with abnormal WBCT20 results indicating VICC, $79 \%$ of those treated with FAV-A showed resolution of coagulopathy after 1 or 2 doses of antivenom, whereas $65 \%$ of those treated with AV-C required 4 or more doses of antivenom to achieve the same result. In another case, a patient reportedly received 30 vials of AV-C over $5 \mathrm{~d}$ without improvement until she was given 8 vials of EchiTAb-Plus-ICP. ${ }^{21}$ According to Dr Roberto Stock (Characterization of antivenoms available in West and Central Africa, unpublished presentation, Fourth African Francophone Congress of Emergency Medicine, June 2012, Cotonou, Benin), sodium dodecyl sulfate polyacrylamide gel electrophoresis and high-performance liquid chromatography analysis identified numerous impurities in Bharat's Asna AV-C and several other Indian-made antivenoms for Africa. This is consistent with the severe pyrogenic reaction documented in the chart of Patient 1 after she received AV-C, which was the only antivenom available at the hospital before the arrival of the authors.

The use of vitamin $\mathrm{K}$ and the antifibrinolytic tranexamic acid, given to Patient 1 before our arrival, was similarly ineffective, which is not surprising given the mechanism of VICC from Echis envenomation. Prothrombin-activating Echis venoms produce deficiencies in multiple clotting factors, and there is no demonstrable role for the use of vitamin $\mathrm{E}$, vitamin $\mathrm{K}$, or antifibrinolytics in the management of VICC from these snakes. ${ }^{8,18,22,23}$ The use of prophylactic antibiotics for snakebite is typically unnecessary because most infections are secondary to tissue destruction or the ill-advised first aid measures commonly employed by traditional healers rather than the bite itself. ${ }^{22}$ Antibiotics were given to Patient 1 because she was initially seen by a local healer who made a series of incisions around the bite and rubbed a mixture of dung and dirt into the wounds.

\section{Conclusions}

Antivenom proved remarkably effective in both cases despite long treatment delays and the serious condition of the patients. Snakebite patients presenting with signs and symptoms indicating the presence of active venom in the bloodstream should receive antivenom regardless of the length of time since the bite or the prior use of antivenom for their current envenomation. Patient 1 made a dramatic recovery from her severe late-stage 
envenomation after receiving Antivipmyn despite the fact that $10 \mathrm{~d}$ had passed since the bite occurred. The striking differences between the samples of CSF taken at $\mathrm{H}_{3}$ vs $\mathrm{H}_{18}$ in Patient 2 and the complete recoveries seen in both cases demonstrate the incredible ability of highquality antivenom to reverse the life-threatening effects of an envenomation, seemingly up to the final moments before death. Conversely, the use of an ineffective lowquality product such as AV-C can cause preventable suffering and a total loss of confidence in antivenom therapy among healthcare professionals and patients alike. It is imperative that we continue efforts to improve the production and distribution of effective antivenoms for the developing world and simultaneously work to reduce the number of dangerous and ineffective products that are currently on the market.

Acknowledgments: This article is dedicated to the staff at the Hôpital Evangelique Bembèrèkè, who work tirelessly to save lives under challenging conditions every day. We recognize their clinical skill and thank them for the generous hospitality shown to our team during the study period.

Author Contributions: Clinical monitoring and assessment (JB, CA, BT); data analysis and interpretation (JB, JPC and AM); manuscript drafting and critical revision for intellectual content (JB and JPC). All authors read and approved the final manuscript.

Financial/Material Support: None.

Disclosures: None.

\section{References}

1. Karaye KM, Mijinyawa MS, Yakasai AM, Kwaghe V, Joseph GA, Iliyasu G, et al. Cardiac and hemodynamic features following snakebite in Nigeria. Int J Cardiol. 2012; 156(3):326-8.

2. Habib AG, Kuznik A, Hamza M, Abdullahi MI, Chedi BA, Chippaux J-P, et al. Snakebite is under appreciated: appraisal of burden from West Africa. PLoS Negl Trop Dis. 2015;9:(9) e0004088.

3. Einterz EM, Bates ME. Snakebite in northern Cameroon: 134 victims of bites by the saw-scaled or carpet viper, Echis ocellatus. Trans R Soc Trop Med Hyg. 2003;97(6): 693-6.

4. Habib AG, Abubakar SB. Factors affecting snakebite mortality in north-eastern Nigeria. Int Health. 2011;3(1):50-5.

5. Warrell DA, Davidson NMcD, Greenwood BM, Ormerod LD, Pope HM, Watkins BJ, et al. Poisoning by bites of the saw-scaled or carpet viper (Echis carinatus) in Nigeria. $Q$ J Med. 1977;46(181):33-62.

6. Fonseka CL, Jeevagan V, Gnanathasan CA. Life threatening intracerebral haemorrhage following saw-scaled viper (Echis carinatus) envenoming -authenticated case report from Sri Lanka. BMC Emerg Med. 2013;13(1):5.

7. Rogalski A, Soerensen C, Brouw den BO, Lister C, Dashvesky D, Arbuckle K, et al. Differential procoagulant effects of saw-scaled viper (Serpentes: Viperidae: Echis) snake venoms on human plasma and the narrow taxonomic ranges of antivenom efficacies. Toxicol Lett. 2017;280: 159-70.

8. Maduwage K, Isbister GK. Current treatment for venominduced consumption coagulopathy resulting from snakebite. PLoS Negl Trop Dis. 2014;8(10):e3220.

9. Berling I, Brown SGA, Miteff F, Levi C, Isbister GK. Intracranial haemorrhages associated with venom induced consumption coagulopathy in Australian snakebites (ASP21). Toxicon. 2015;102(C):8-13.

10. Benjamin JM, Chippaux J-P, Sambo BT, Massougbodji A. Delayed double reading of whole blood clotting test (WBCT) results at 20 and 30 minutes enhances diagnosis and treatment of viper envenomation. J Ven Anim Toxins Incl Trop Dis. 2018;24(1):38.

11. Chippaux JP, Baldé MC, Sessinou É, Yéro Boiro M, Massougbodji A. Evaluation of a new polyvalent antivenom against snakebite envenomation (Inoserp ${ }^{\circledR}$ Panafricain) in two different epidemiological settings: Northern Benin and Maritime Guinea [in French]. Med Sante Trop. 2015;25 (1):56-64.

12. Chippaux JP, Massougbodji A, Stock RP, Alagon A. Clinical trial of an $\mathrm{F}(\mathrm{ab}$ ') 2 polyvalent equine antivenom for African snake bites in Benin. Am J Trop Med Hyg. 2007;77(3):538-46

13. Sandbank U, Jerushalmy Z, Ben-David E, De Vries A. Effect of Echis coloratus venom on brain vessels. Toxicon. 1974;12(3):267-71.

14. Boyer LV, Seifert SA, Clark RF, McNally JT, Williams SR, Nordt SP, et al. Recurrent and persistent coagulopathy following pit viper envenomation. Arch Intern Med. 1999;159(7):706-10.

15. Shah KH, Edlow JA. Distinguishing traumatic lumbar puncture from true subarachnoid hemorrhage. J Emerg Med. 2002;23(1):67-74.

16. Czuczman AD, Thomas LE, Boulanger AB, Peak DA, Senecal EL, Brown DF, et al. Interpreting red blood cells in lumbar puncture: distinguishing true subarachnoid hemorrhage from traumatic tap. Acad Emerg Med. 2013;20 (3):247-56.

17. Perry JJ, Alyahya B, Sivilotti MLA, Bullard MJ, Emond M, Sutherland J, et al. Differentiation between traumatic tap and aneurysmal subarachnoid hemorrhage: prospective cohort study. BMJ. 2015;350:h568.

18. Habib AG, Warrell DA. Antivenom therapy of carpet viper (Echis ocellatus) envenoming: effectiveness and strategies for delivery in West Africa. Toxicon. 2013;69:82-9.

19. Visser LE, Kyei-Faried S, Belcher DW, Geelhoed DW, Leeuwen JSV, van Roosmalen J. Reply to comment on: Failure of a new antivenom to treat Echis ocellatus snake bite in rural Ghana: the importance of quality surveillance. Trans R Soc Trop Med Hyg. 2009;103(9):964-5.

20. Visser LE, Kyei-Faried S, Belcher DW, Geelhoed DW, van Leeuwen JS, van Roosmalen J. Failure of a new antivenom to treat Echis ocellatus snake bite in rural Ghana: the importance of quality surveillance. Trans $R$ Soc Trop Med Hyg. 2008;102(5):445-50. 
21. Akintayo RO, Yusuf IA. Severe snakebite envenomation: a near-fatal case of probable antivenom inefficacy. Sav J Med Res Pract. 2015;4(1):26.

22. Blaylock RS. The identification and syndromic management of snakebite in South Africa. SA Fam Pract. 2005;47 (9):48-53.
23. Howes JM, Kamiguti AS, Theakston RDG, Wilkinson MC, Laing GD. Effects of three novel metalloproteinases from the venom of the West African saw-scaled viper, Echis ocellatus on blood coagulation and platelets. Biochim Biophys Acta Gen Subj. 2005;1724(1-2): 194-202. 